\title{
Malaria grave en mujeres gestantes hospitalizadas entre el 2010 y el 2014 en el departamento de Antioquia, Colombia
}

\author{
Alberto Tobón-Castaño, John Edison Betancur \\ Grupo de Malaria, Facultad de Medicina, Universidad de Antioquia, Medellín, Colombia
}

Introducción. La malaria (o paludismo) durante la gestación impacta negativamente la salud de la madre y del neonato, con alto riesgo de complicaciones clínicas y mortalidad. En las regiones de alta endemia se han caracterizado, especialmente, la anemia materna y el bajo peso al nacer, pero es poco conocido el espectro clínico en las zonas de baja endemia. Objetivo. Caracterizar clínica y epidemiológicamente los episodios de malaria en mujeres gestantes hospitalizadas en el departamento de Antioquia entre el 2010 y el 2014.

Materiales y métodos. Se hizo un estudio descriptivo, transversal y retrospectivo, con historias clínicas de mujeres gestantes con malaria por Plasmodium falciparum y $P$. vivax. Se utilizaron los criterios diagnósticos de malaria complicada de la Organización Mundial de la Salud (OMS) y de la Guía para la atención clínica integral del paciente con malaria vigente en Colombia.

Resultados. Se analizaron 111 casos; el 13,5 \% se clasificó como complicación grave según los criterios de la OMS, porcentaje que ascendió a 23,4 \% según los criterios de la guía colombiana. Las complicaciones detectadas fueron disfunción hepática, anemia, acidosis y trombocitopenia grave. No se observó diferencia en la frecuencia de las complicaciones según la especie de plasmodio. El 39,4\% de los casos presentó signos generales de peligro; la palidez y la ictericia fueron los más frecuentes. El 40,5\% presentó signos de peligro para la gestación como la cefalea persistente, el dolor abdominal y el sangrado vaginal.

Conclusiones. La malaria grave se presenta con gran frecuencia en las mujeres gestantes, sin diferencia según la especie de plasmodio, y se manifiesta con signos de peligro precozmente reconocibles. Se encontró un subregistro hospitalario del $88 \%$ de los casos graves y falta de exámenes de laboratorio para un diagnóstico más completo. Se requiere un protocolo para el diagnóstico clínico de las mujeres gestantes con malaria.

Recibido: 07/03/18

Aceptado: $07 / 10 / 18$

Publicado: 09/10/18

Citación:

Tobón-Castaño A, Betancur JE. Malaria grave en mujeres gestantes hospitalizadas entre el 2010 y el 2014 en el departamento de Antioquia, Colombia. Biomédica. 2019;39:354-69.

https://doi.org/10.7705/biomedica.v39i3.4403

\section{Correspondencia:}

Alberto Tobón-Castaño, Sede Investigación Universitaria, Universidad de Antioquia, Calle $62 \mathrm{~N}^{\circ}$ 52-59, laboratorio 610, Medellín, Colombia Telefax: (574) 2196487

alberto.tobon1@udea.edu.co

\section{Contribución de los autores:}

John Edison Betancur: recolección de datos

Ambos autores participaron en el diseño del estudio, el análisis de los datos y la escritura del manuscrito.

Financiación:

Colciencias, Gobierno de Colombia, Programa de Jóvenes Investigadores, Convocatoria 645 de 2014, que aportó los recursos para la participación de John Edison Betancur Romero, y Grupo de Malaria y Estrategia de sostenibilidad de la Vicerrectoría de Investigación 2015-2016, Universidad de Antioquia.

\section{Conflicto de intereses:}

Los autores declaramos no tener conflicto de

intereses para la publicación de este manuscrito.
Palabras clave: Plasmodium vivax, Plasmodium falciparum; malaria; embarazo; Colombia.

\section{Severe malaria in pregnant women hospitalized between 2010 and 2014 in the Department of Antioquia (Colombia)}

Introduction: Malaria during pregnancy has a negative impact on maternal-neonatal health, with a high risk of clinic complications and mortality. High endemic areas are specially characterized by maternal anaemia and low birth weight. The clinical spectrum is little known in low endemic areas.

Objective: To clinically and epidemiologically characterize malaria episodes in hospitalized pregnant women in the Department of Antioquia (Colombia) in the period 2010-2014. Materials and methods: Retrospective, cross-sectional, descriptive study with medical records of pregnant women with $P$. falciparum and $P$. vivax malaria. The WHO severe malaria diagnostic criteria and the Colombian Guía para la atención clínica integral del paciente con malaria (guidelines for comprehensive malaria treatment) were used. Results: We analyzed 111 cases, out of which $13.5 \%$ were classified as severe malaria according to the WHO criteria. Following the Colombian Guidelines, the proportion increased to $23.4 \%$. Identified complications included hepatic dysfunction, anaemia, acidosis, and severe thrombocytopenia. No difference in the frequency of complications by Plasmodium species was observed; $39.4 \%$ of the cases presented general danger signs, pallor and jaundice being the most frequent; $40.5 \%$ showed danger signs for pregnancy, such as persistent headache, abdominal pain, and vaginal bleeding.

Conclusions: Severe malaria is a highly frequent event in pregnant women, without differences by Plasmodium species. It shows early recognizable dangers signs. Hospital under-reporting was identified in $88 \%$ of severe cases as well as a lack of laboratory tests for a more comprehensive diagnosis. A protocol for the clinical diagnosis of pregnant women with malaria is required.

Keywords: Plasmodium vivax; Plasmodium falciparum; malaria; pregnancy; Colombia. 
La malaria, o paludismo, es un reto vigente para la salud pública mundial y nacional. En el 2016 la Organización Mundial de la Salud (OMS) estimó que en el mundo ocurrieron 216 millones de casos y 445.000 muertes; el $90 \%$ de la carga mundial se ubica en el continente africano, en donde las mujeres embarazadas y los niños menores de cinco años aportan la mayor mortalidad (1).

Se calcula que 125 millones de embarazos ocurren anualmente en las regiones donde existe transmisión autóctona de Plasmodium falciparum y $P$. vivax (2). De estas mujeres gestantes, cada año cerca de 25 millones se encuentran expuestas a infecciones por malaria en áreas donde $P$. vivax es endémico (3). Los efectos de $P$. falciparum se han caracterizado en las áreas de alta transmisión, como África, en donde esta especie es responsable de una gran proporción de morbilidad y mortalidad materna y neonatal (4). A la malaria gestacional se le han atribuido del 3 al $15 \%$ de los casos de anemia materna, del 8 al $14 \%$ de los de bajo peso al nacer, y del 3 al $8 \%$ de los de mortalidad infantil ocurridos en las zonas de alta endemia. Además, se la ha asociado con 75.000 a 200.000 muertes infantiles ocurridas anualmente en África, así como con una tasa de mortalidad perinatal de 25 a 80 por 1.000 nacidos vivos $(5,6)$.

En las mujeres gestantes la presentación clínica del paludismo es diferente y más grave. En ellas, se ha estimado un riesgo tres veces mayor de complicaciones $(3,7)$. En las zonas hiperendémicas y con transmisión estable, la adquisición de inmunidad en las etapas tempranas de la vida hace que muchas de estas infecciones cursen de forma asintomática durante el embarazo (8). Mientras tanto, en las zonas de endemia baja o media, o con transmisión inestable o estacional, son frecuentes las manifestaciones clínicas y se ha estimado una mayor probabilidad de complicaciones, como malaria cerebral, hipoglucemia, dificultad respiratoria y anemia hemolítica (7). En comparación con las regiones de África con alta endemia y prevalencia de $P$. falciparum, en las regiones endémicas de Latinoamérica, donde prevalecen las infecciones por $P$. vivax, poco se ha estudiado la expresión clínica de la malaria en mujeres embarazadas (9).

Entre los efectos más reconocidos de la malaria gestacional se incluyen el parto prematuro, el bajo peso al nacer y la anemia materna grave que, además, se han sido asociado con la presencia de Plasmodium spp. en la placenta (10). Los efectos de las infecciones por $P$. vivax en el embarazo $y$ en el neonato se han estudiado poco, y el problema reviste especial importancia porque, en el caso de esta infección, las mujeres gestantes reciben esquemas de tratamiento parciales para evitar los efectos secundarios sobre el feto, lo que incrementa la probabilidad de recaídas con múltiples episodios durante el embarazo (9). Aunque con menor frecuencia que en las infecciones por $P$. falciparum, se ha informado enfermedad grave, parto prematuro, retardo del crecimiento intrauterino, abortos y mortinatos en aquellas producidas por $P$. vivax (10-12).

En Colombia un estudio prospectivo y descriptivo de 166 mujeres con diagnóstico de malaria durante el control prenatal reveló que $15(9 \%)$ de los casos estudiados se clasificaron como malaria grave según los criterios de la OMS (7), $10(67 \%)$ correspondieron a infecciones producidas por $P$. vivax y 5 (33\%), por P. falciparum (13).

La investigación sobre malaria gestacional en las zonas de baja endemia es escasa, sobre todo en los centros hospitalarios de mediana y alta complejidad; no se conocen las tasas de hospitalización, ni la letalidad, ni 
las características clínicas de las mujeres gestantes con cuadros de gravedad. Esta información es fundamental para la comprensión de la enfermedad en las mujeres gestantes y para conocer la aplicabilidad en ellas de los criterios establecidos por la OMS para la malaria grave, con el fin de emplear protocolos de atención adaptados a las condiciones epidemiológicas de regiones de baja endemia y con transmisión inestable de malaria, como es el caso de Colombia.

El propósito de este estudio fue caracterizar clínica y epidemiológicamente los episodios de malaria en mujeres gestantes hospitalizadas en el departamento de Antioquia entre 2010 y 2014.

\section{Materiales y métodos}

Se llevó a cabo un estudio descriptivo de corte transversal y retrospectivo para caracterizar los episodios de malaria gestacional en el ámbito hospitalario. Esto se hizo en el departamento de Antioquia, segundo en Colombia en densidad poblacional (6.378.132 habitantes), donde se presentaron 9.277 casos de malaria (22,8\% de los 40.768 casos nacionales) en el 2014 , con un índice parasitario anual (IPA) de 145,5 por 100.000 habitantes.

La información se recolectó en tres hospitales de tercer nivel de Medellín, los cuales sirven como centros de recepción de pacientes, y en los hospitales de segundo nivel en las tres subregiones que son endémicas para malaria: Urabá ( 1.538 casos; IPA=239,3), Bajo Cauca $(5.578$ casos; IPA=1.901,5) y Magdalena Medio (80 casos; IPA=70,2) (14).

Se revisaron los registros clínicos hospitalarios y los del Sistema Nacional de Vigilancia Epidemiológica (Sivigila) de las mujeres gestantes con diagnóstico de malaria durante el periodo de 2010 a 2014. La población de referencia estuvo constituida por las mujeres embarazadas atendidas en seis hospitales de referencia del departamento de Antioquia: Hospital Universitario San Vicente Fundación, Hospital Pablo Tobón Uribe y Hospital General de Medellín, en la capital; E.S.E. Hospital César Uribe Piedrahita, en el municipio de Caucasia; Hospital Antonio Roldán Betancur, en el municipio de Apartadó, y Hospital La Cruz E.S.E., en el municipio de Puerto Berrío.

Se incluyeron mujeres con cualquier edad gestacional, con gota gruesa positiva para cualquier especie de Plasmodium según los registros hospitalarios, y que hubieran estado hospitalizadas por lo menos durante 24 horas en alguno de los hospitales donde se desarrolló el estudio. Además, se buscaron las historias con diagnóstico de malaria según la CIE-10.

Para la recolección de los datos, se utilizó un cuestionario que permitió extraer información de la historia clínica de las mujeres gestantes sobre aspectos demográficos, clínicos (laboratorio, signos y síntomas) y epidemiológicos del diagnóstico, así como sobre el tratamiento y el seguimiento del episodio de malaria. Se emplearon los criterios clínicos de complicación de la OMS (15) y los de la 'Guía para la atención clínica integral del paciente con malaria' de Colombia (16). Los casos se clasificaron como: complicación grave, complicación moderada o sin complicaciones. Se determinó la presencia de signos de peligro, clínicos o parasitológicos, según los criterios propuestos por Tobón (17).

Los datos demográficos, clínicos y epidemiológicos se almacenaron en una base de datos en Microsoft Access ${ }^{\mathrm{TM}}$, y se procesaron mediante el paquete estadístico SPSS ${ }^{\mathrm{TM}}$, versión 24 para Windows, licenciados para la Universidad de Antioquia. 
Se utilizó la estadística descriptiva para establecer las características demográficas, las características clínicas de la infección y los resultados de laboratorio clínico. Para las variables continuas, se calcularon la media, la mediana, la desviación estándar, los intervalos de confianza y el rango intercuartílico (según la distribución de los datos); para las variables cualitativas, se estimaron las frecuencias absolutas y los porcentajes. Se usaron la prueba t de Student, la U de Mann-Whitney y la de ji al cuadrado de Pearson para establecer las diferencias entre los resultados clínicos, los resultados de los exámenes de laboratorio, el tipo de infección, los signos de peligro y las complicaciones. Se estimó el Odds Ratio (OR) y su intervalo del confianza (IC) del $95 \%$ para comparar proporciones entre las variables cualitativas.

\section{Aspectos éticos}

Se respetaron las normas éticas internacionales para investigación biomédica con sujetos humanos establecidas por la OMS y las normas del Ministerio de Protección Social de Colombia (Resolución 8430 de 1993).

Para acceder a los registros clínicos, se obtuvo el aval del Comité de Ética del Hospital Universitario San Vicente Fundación (24 de junio de 2015), el del Hospital Pablo Tobón Uribe (Acta 14 de 2015) y el del Hospital General de Medellín (Acta 7 de 2015). Además, se contó con el aval de la dirección del E.S.E. Hospital César Uribe Piedrahita, la del Hospital Antonio Roldán Betancur y la del Hospital La Cruz E.S.E., y el del Instituto de Investigaciones Médicas de la Universidad de Antioquia.

\section{Resultados}

\section{Características sociodemográficas}

Se revisaron 389 historias clínicas hospitalarias de mujeres embarazadas con enfermedad febril y sospecha de malaria, de las cuales 111 cumplían el criterio de diagnóstico confirmado por gota gruesa durante la hospitalización ( $n=109)$, o cuyo diagnóstico se informó en la remisión $(n=2)$. La mayoría [n=68, $(61,3 \%)$ ] de las pacientes fueron atendidas en hospitales de tercer nivel de complejidad de Medellín, 26,1 \% ( $n=29)$ en el municipio de Caucasia, 13, 5\% ( $n=15)$, en Apartadó y, 5,4\% $(n=6)$, en Puerto Berrío, en hospitales de segundo nivel.

La edad de las pacientes estuvo entre los 13 y los 43 años (media=22,7; desviación estándar, $\mathrm{DE}=6,01$; mediana $=21$ ); el $57,8 \%$ se encontraba entre los 13 y los 20 años. El grupo étnico se informó en 46 gestantes y en $61 \%$ de ellas correspondía a mestizas. La residencia se conoció en 81 de ellas, de las cuales el $49 \%$ residía en la zona rural. Del total estudiado (111), 97 (87\%) estaban afiliadas al Sistema General de Seguridad Social en Salud (SGSSS) y 86 (78\%) pertenecían al régimen subsidiado (cuadro 1).

\section{Motivo de consulta}

El 46,8 \% de las pacientes ingresó al servicio de urgencias, el 37,8 \% fue remitido para hospitalización y el 15,3\% correspondió a hospitalizadas en el momento del diagnóstico. El promedio de evolución de la enfermedad actual fue de 4,3 días $(\mathrm{DE}=4,5)$. Los síntomas más frecuentes desde el inicio de la enfermedad fueron la fiebre $(79,3 \%)$ y la cefalea $(67,6 \%)$; la tríada de fiebre, cefalea y escalofríos se presentó en el $41 \%$ de casos. Estos y otros síntomas referidos desde el inicio de la enfermedad se presentan en la figura 1. 
Cuadro 1. Características sociodemográficas en mujeres embarazadas con malaria en Antioquia según la condición clínica, 2010-2014

\begin{tabular}{|c|c|c|c|c|c|c|c|}
\hline \multirow{3}{*}{ Característica } & \multicolumn{6}{|c|}{ Condición clínica de la malaria } & \multirow{3}{*}{$\mathbf{p}^{*}$} \\
\hline & \multicolumn{2}{|c|}{ Total } & \multicolumn{2}{|c|}{ No complicada } & \multicolumn{2}{|c|}{ Complicada } & \\
\hline & $n=111$ & $(\%)$ & $\mathrm{n}=85$ & (\%) & $n=26$ & $(\%)$ & \\
\hline Edad: años / M (DE) & $23(6)$ & & $23(7)$ & & $23(6)$ & & $0,845^{\star *}$ \\
\hline Edad: años / Me (RIC) & $21(18-26)$ & & $21(18-27)$ & & $21(18-26)$ & & \\
\hline \multicolumn{8}{|l|}{ Edad en años } \\
\hline 0 a 22 & $63 / 109$ & $(57,8)$ & $48 / 83$ & $(57,8)$ & $15 / 26$ & $(57,7)$ & 0,99 \\
\hline 23 a 50 & 46 & $(42,2)$ & 35 & $(42,2)$ & 11 & $(42,3)$ & \\
\hline \multicolumn{8}{|l|}{ Etnia } \\
\hline Negra & $10 / 46$ & $(21,7)$ & $8 / 36$ & $(22,2)$ & $2 / 10$ & $(20,0)$ & 0,488 \\
\hline Indígena & $8 / 46$ & $(17,4)$ & $5 / 36$ & $(13,9)$ & $3 / 10$ & $(30,0)$ & \\
\hline Mestiza & $28 / 46$ & $(60,9)$ & $23 / 36$ & $(63,9)$ & $5 / 10$ & $(50,0)$ & \\
\hline \multicolumn{8}{|l|}{ Zona de residencia } \\
\hline Urbana & $41 / 81$ & $(50,6)$ & $35 / 64$ & $(54,7)$ & $6 / 17$ & $(35,3)$ & 0,155 \\
\hline Rural & $40 / 81$ & $(49,4)$ & $29 / 64$ & $(45,3)$ & $11 / 17$ & $(64,7)$ & \\
\hline \multicolumn{8}{|l|}{ Afiliación en salud } \\
\hline No & $14 / 111$ & $(12,6)$ & $13 / 85$ & $(15,3)$ & $1 / 26$ & $(3,8)$ & 0,124 \\
\hline Sí & 97 & $(87,4)$ & 72 & $(84,7)$ & $25 / 26$ & $(96,2)$ & \\
\hline \multicolumn{8}{|l|}{ Tipo de afiliación } \\
\hline Vinculado & $10 / 111$ & $(9,0)$ & $10 / 85$ & $(11,8)$ & $0 / 26$ & $(0,0)$ & 0,428 \\
\hline Subsidiado & 86 & $(77,5)$ & 64 & $(75,3)$ & 22 & $(84,6)$ & \\
\hline Contributivo & 11 & $(9,9)$ & 8 & $(9,4)$ & 3 & $(11,5)$ & \\
\hline Ninguno & 3 & $(2,7)$ & 2 & $(2,4)$ & 1 & $(3,8)$ & \\
\hline Particular & 1 & $(0,9)$ & 1 & $(1,2)$ & 0 & $(0,0)$ & \\
\hline
\end{tabular}

M: media; Me: mediana; DE: desviación estándar; RIC: rango intercuartílico

* Prueba de ji al cuadrado de independencia; significación del 0,05

** Prueba U de Mann-Whitney; significación del 0,05

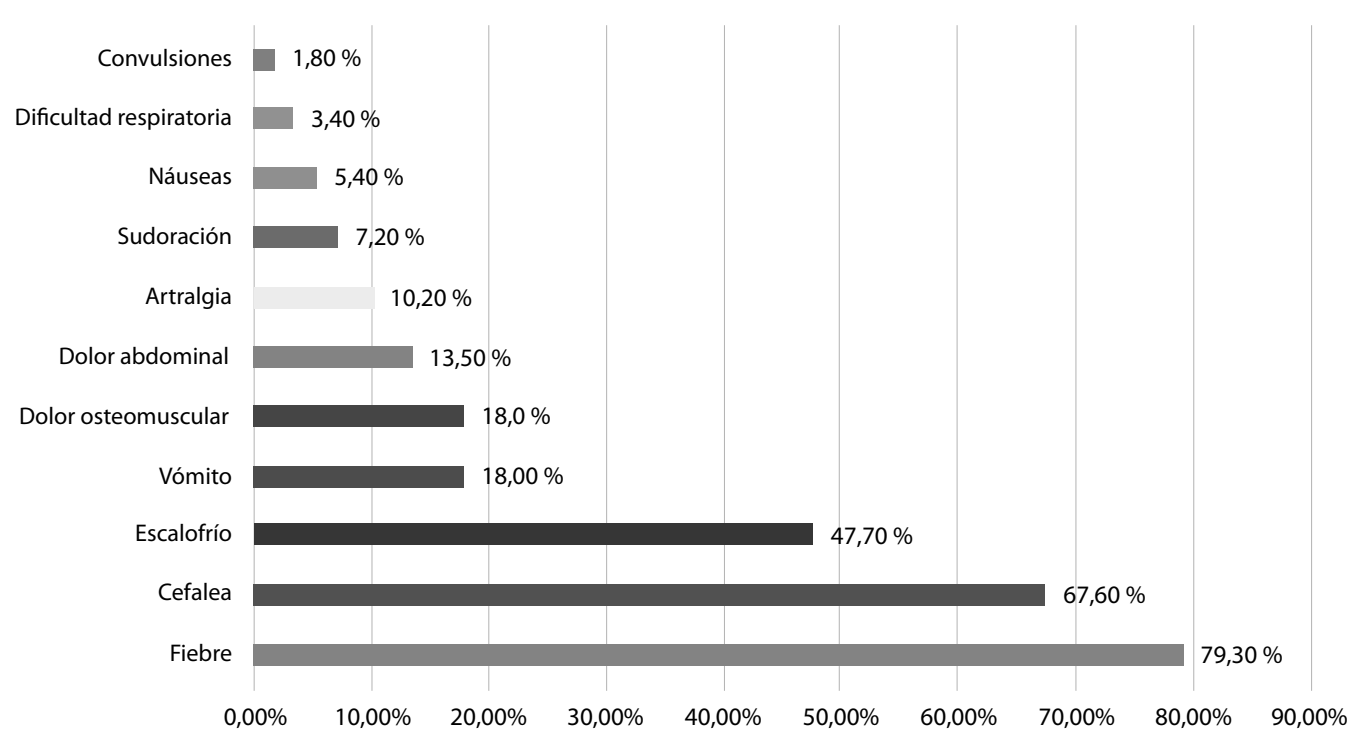

Figura 1. Síntomas en 111 mujeres gestantes con diagnóstico de malaria, Antioquia, 2010-2014 
No hubo diferencias con respecto a la presencia de fiebre entre los casos de $P$. vivax $(81,1 \%$ y los de $P$. falciparum $(81,2 \%)$ ( $p=0,994)$; lo mismo sucedió con la cefalea $(69,4$ Vs. $68,7 \%)(p=0,418)$. Los valores de la mediana de los signos vitales al ingreso fueron: temperatura de $37,0{ }^{\circ} \mathrm{C}$ (rango intercuartílico, RIC=36,0-38,5), tensión arterial sistólica de $110 \mathrm{~mm}$ $\mathrm{Hg}(\mathrm{RIC}=100-120)$, tensión diastólica de $68 \mathrm{~mm} \mathrm{Hg}(\mathrm{RIC}=60-72)$ y frecuencia respiratoria de 19 por minuto $(\mathrm{RIC}=18-20)$; el promedio del pulso fue de 88 latidos por minuto (desviación estándar, $D E=18,3$ ). No se encontraron diferencias en las medianas de los signos vitales según la especie parasitaria.

\section{Antecedentes ginecoobstétricos, de enfermedad general y de malaria}

La edad gestacional al momento del diagnóstico de malaria se estableció en 108 mujeres embarazadas, y estuvo entre 6,4 y 39,6 semanas (media=27,2; DE=9,6; mediana=29,8). En 84 mujeres gestantes se estableció por ecografía y, en las restantes, según la fecha de la última menstruación. Según su valor, 14 (13\%) se encontraban en el primer trimestre de gestación, 24 (22\%) en el segundo y 70 (65\%) en el tercero.

En el momento del diagnóstico de malaria, las mujeres gestantes relataron haber asistido, en promedio, a 3,7 visitas de control prenatal $(\mathrm{DE}=2,1$; rango=1-9; mediana=3,0). El 71,2 \% reportó por lo menos un embarazo previo y, el $22,4 \%$, más de tres embarazos. El promedio de hijos nacidos vivos fue de 2,7 ( $\mathrm{DE}=2,4)$ y el de abortos, de 1,2 (DE=0,7).

Solo en 24 casos se registraron los antecedentes de malaria antes de la gestación; 2 presentaron un episodio de malaria por $P$. falciparum y 22 por $P$. vivax ( 8 con un antecedente, 8 con dos antecedentes y 6 con más de dos). Diez mujeres gestantes habían presentado, al menos, un episodio de malaria por $P$. vivax en el último año.

\section{Diagnóstico de malaria}

El diagnóstico registrado en la historia clínica según la CIE-10 (101 casos), se presenta en el cuadro 2, donde se contrasta con la clasificación basada en los resultados de laboratorio. Con el examen de gota gruesa se

Cuadro 2. Código de la ClE-10 en 101 de los 111 casos de malaria en gestantes y clasificación del estado clínico según los hallazgos de laboratorio*

\begin{tabular}{lcccc}
\multicolumn{5}{c}{ Casos complicados, según laboratorio clínico } \\
& P. falciparum & P. vivax & Mixta & Total \\
\hline B508 P. falciparum complicado & 1 & 0 & 0 & 1 \\
B518 P. vivax complicado & 0 & 2 & 0 & 2 \\
B519 P. vivax no complicado & 0 & 4 & 0 & 4 \\
B54 Paludismo no especificado & 4 & 13 & 1 & 18 \\
Total & 5 & 19 & 1 & 25 \\
\multicolumn{5}{c}{ Casos no complicados, según laboratorio clínico } \\
B508 P. falciparum complicado & $\boldsymbol{P}$ falciparum & $\boldsymbol{P}$. vivax & Mixta & Total \\
B509 P. falciparum no complicado & 0 & 1 & 0 & 1 \\
B518 P. vivax complicado & 2 & 2 & 1 & 5 \\
B519 P. vivax no complicado & 0 & 1 & 0 & 1 \\
B54 Paludismo no especificado & 0 & 5 & 0 & 5 \\
O98 Otras infecciones & 6 & 53 & 2 & 61 \\
Total & 1 & 2 & 0 & 3 \\
\hline
\end{tabular}

* Un caso complicado y tres no complicados no tienen código CIE-10; los otros seis casos tienen código CIE-10, pero no se identificó la especie en la historia clínica. 
diagnosticó la infección por $P$. vivax en 85 (77,0 \%) mujeres gestantes, por $P$. falciparum en $16(15,2 \%)$ y la infección mixta por estas especies en $4(3,8$ $\%)$; en 6 no se informó la especie.

El recuento parasitario se informó en 52 (46,8 \%) diagnósticos. Entre los casos de $P$. vivax, el promedio fue de 10.331 parásitos/ $\mu \mathrm{l}(\mathrm{DE}=14.685$; mediana 5.800; RIC=2.480-14.000), el 67,4\% (29 de 43) con recuentos inferiores a 10.000 anillos/ $\mu \mathrm{l}$ y un caso (2,4\%) por encima de los 50.000 parásitos/ $\mu \mathrm{l}$. Entre los casos de $P$. falciparum, el promedio del recuento

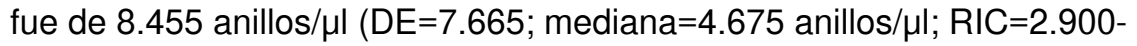
16.240) y ningún recuento fue superior a 20.000 parásitos/ $\mu$. El promedio del recuento en las infecciones mixtas fue de 3.467 parásitos/ $\mu \mathrm{l}(\mathrm{DE}=3.788$; mediana=1.360; $\mathrm{RIC}=1.200-7.840$ ) y todos fueron inferiores a 8.000 .

\section{Resultados de laboratorio}

En el cuadro 3 se presentan los resultados de los exámenes de laboratorio usados para clasificar las complicaciones clínicas y en el cuadro 4, los valores de referencia para la clasificación del estado de gravedad.

A 109 mujeres gestantes (cuadro 4) se les practicó hemograma durante el episodio de malaria y el promedio de la hemoglobina $(\mathrm{Hb})$ fue de $10,2 \mathrm{~g} / \mathrm{dl}$ $(\mathrm{DE}=1,9) ; 91(83,5 \%)$ mujeres gestantes presentaron algún grado de anemia $(\mathrm{Hb}<12 \mathrm{~g} / \mathrm{dl})$ y, $4(3,6 \%)$, anemia grave $(\mathrm{Hb}<7 \mathrm{~g} / \mathrm{dl})$; el menor valor de la hemoglobina fue de $3,6 \mathrm{~g} / \mathrm{dl}$, reportado en una mujer embarazada infectada con $P$. vivax.

El recuento de plaquetas se hizo en 102 casos, con una mediana de 156.500 plaquetas/ $\mu \mathrm{l}(\mathrm{RIC}=70.000$ - 220.000); 57 (55,8\%) mujeres embarazadas presentaron trombocitopenia (menos de 150.000 plaquetas/ $\mu \mathrm{l}$ ), en $13(12,7 \%)$ de ellas la trombocitopenia fue grave (menos de 50.000 plaquetas $/ \mu \mathrm{l})$. Solo se presentaron diferencias estadísticamente significativas entre las mujeres embarazadas infectadas con $P$. vivax y las mujeres infectadas por $P$. falciparum en los niveles de hemoglobina $(10,3 \mathrm{Vs}$. 8,9 $\mathrm{g} / \mathrm{dl}$; $\mathrm{p}=0,004)$.

En $43(38,7 \%)$ embarazadas se midió la creatinina, cuya mediana fue de $0,6 \mathrm{mg} / \mathrm{dl}(\mathrm{RIC}=0,5-0,7)$; dos $(4,6 \%)$ tuvieron cifras por encima de $1 \mathrm{mg} / \mathrm{dl}$. El nitrógeno ureico sanguíneo (BUN) se midió en 21 casos; la mediana fue de 8,0 $\mathrm{mg} / \mathrm{dl}(\mathrm{RIC}=5,0-10,0)$ y solo una paciente $(4,7 \%)$ presentó un valor elevado $(35,9 \mathrm{mg} / \mathrm{dl})$. No se encontraron diferencias en estos marcadores según la especie de Plasmodium. La bilirrubina sérica total se midió en 42 mujeres

Cuadro 3. Resultados de laboratorio por especie de plasmodio en mujeres gestantes con malaria en Antioquia, 2010-2014

\begin{tabular}{|c|c|c|c|c|c|c|c|c|c|}
\hline \multirow{2}{*}{ Examen de laboratorio } & \multicolumn{3}{|c|}{ Plasmodium falciparum $(\mathrm{n}=16)$} & \multicolumn{3}{|c|}{ Plasmodium vivax $(\mathrm{n}=85)$} & \multicolumn{3}{|c|}{ Infección mixta $(n=4)$} \\
\hline & $\mathbf{n}$ & Mediana & Q1/Q3 & $\mathbf{n}$ & Mediana & Q1/Q3 & $\mathbf{n}$ & Mediana & Q1/Q3 \\
\hline Hemoglobina (M-DE) (g/dl) & 16 & 8,9 & 1,6 & 83 & 10,3 & 1,9 & 4 & 10,3 & 1,9 \\
\hline Plaquetas (M-DE) (por $\left.\mathrm{mm}^{3}\right)$ & 15 & 163.700 & 106.900 & 77 & 146.100 & 87.500 & 4 & 122.300 & 136.200 \\
\hline Creatinina (mg/dl) & 7 & 0,6 & $(0,5-0,9)$ & 29 & 0,6 & $(0,5-0,6)$ & 2 & 0,6 & $(0,5-0,6)$ \\
\hline Nitrógeno ureico (M-DE) (mg/dl) & 6 & 11,1 & 12,9 & 11 & 7,6 & 3,5 & 1 & 10,0 & - \\
\hline Bilirrubina total (mg/dl) & 10 & 0,9 & $(0,7-15)$ & 27 & 1,0 & $(0,8-1,8)$ & 2 & 1,6 & $(1,5-17)$ \\
\hline Bilirrubina directa (M-DE) (mg/dl) & 9 & 0,6 & 0,6 & 22 & 0,9 & 0,9 & 2 & 1,0 & 0,1 \\
\hline Alanina aminotransferasa (UI/L) & 3 & 16,0 & $(12,3-40,9)$ & 15 & 19,3 & $(12,0-48,0)$ & 2 & 21,3 & $(13,5-29,0)$ \\
\hline Glucemia $(g / d l)$ & 3 & 76,0 & $(68,0-88,0)$ & 13 & 91,0 & $(79,0-105,0)$ & 1 & 97,0 & - \\
\hline $\begin{array}{l}\text { Carga parasitaria (formas } \\
\text { asexuales/microlitro) }\end{array}$ & 6 & 4.675 & $(2.900-16.240$ & 43 & 5.800 & $(2.480-14.000)$ & 3 & 1.360 & $(1.200-7.840)$ \\
\hline
\end{tabular}


Cuadro 4. Clasificación de los resultados de laboratorio según el grado de alteración en mujeres gestantes con malaria en Antioquia, 2010-2014

\begin{tabular}{|c|c|c|c|c|c|c|c|c|}
\hline \multirow{3}{*}{ Examen de laboratorio } & \multicolumn{8}{|c|}{ Grado de alteración } \\
\hline & \multicolumn{2}{|c|}{ Sin alteración } & \multicolumn{2}{|c|}{ Leve } & \multicolumn{2}{|c|}{ Moderada* } & \multicolumn{2}{|c|}{ Grave $^{\star \star}$} \\
\hline & $\mathbf{n}$ & $\%$ & $\mathbf{n}$ & $\%$ & $\mathbf{n}$ & $\%$ & $\mathbf{n}$ & $\%$ \\
\hline Hemoglobina (g/dl) & 18 & 16,5 & 58 & 53,2 & 29 & 26,6 & 4 & 3,7 \\
\hline Valor de referencia & \multicolumn{2}{|c|}{$>12$} & \multicolumn{2}{|c|}{$9,1-12$} & \multicolumn{2}{|c|}{$7-9$} & \multicolumn{2}{|c|}{$<7$} \\
\hline Plaquetas (por mm3) & 45 & 44,1 & 41 & 40,2 & 13 & 12,7 & 3 & 2,9 \\
\hline Valor de referencia & \multicolumn{2}{|c|}{$>150.000$} & \multicolumn{2}{|c|}{$50.000-150.000$} & \multicolumn{2}{|c|}{$25.000-50.000$} & \multicolumn{2}{|c|}{$<25.000$} \\
\hline Bilirrubina total (mg/dl) & 21 & 50,0 & 9 & 21,4 & 6 & 14,3 & 6 & 14,3 \\
\hline Valor de referencia & \multicolumn{2}{|c|}{ Hasta 1,0 } & \multicolumn{2}{|c|}{$1,1-1,5$} & \multicolumn{2}{|c|}{$1,6-3,0$} & \multicolumn{2}{|c|}{$>3,0$} \\
\hline AST (UI/L) & 14 & 70,0 & 4 & 20,0 & - & - & 2 & 10,0 \\
\hline Valor de referencia & \multicolumn{2}{|c|}{$10-35$} & \multicolumn{2}{|c|}{$36-80$} & \multicolumn{2}{|c|}{$81-120$} & \multicolumn{2}{|c|}{$>120$} \\
\hline ALT (UI/L) & 16 & 80,0 & 2 & 10.0 & - & - & 2 & 10,0 \\
\hline Valor de referencia & \multicolumn{2}{|c|}{$9-43$} & \multicolumn{2}{|c|}{$44-80$} & \multicolumn{2}{|c|}{$81-120$} & \multicolumn{2}{|c|}{$>120$} \\
\hline Creatinina (mg/dl) & 41 & 95,3 & 2 & 4,6 & - & - & - & - \\
\hline Valor de referencia & \multicolumn{2}{|c|}{$0,5-1,0$} & \multicolumn{2}{|c|}{$1,1-1,5$} & \multicolumn{2}{|c|}{$1,6-3,0$} & \multicolumn{2}{|c|}{$>3,0$} \\
\hline Nitrógeno ureico (mg/dl) & 20 & 95,2 & 1 & 4,8 & - & - & - & - \\
\hline Valor de referencia & \multicolumn{2}{|c|}{0 a 20} & \multicolumn{2}{|c|}{21 a 40} & \multicolumn{2}{|c|}{41 a 60} & \multicolumn{2}{|c|}{$>60$} \\
\hline
\end{tabular}

AST: aspartato aminotransferasa; ALT: alanino aminotransferasa

* Por encima de este rango se considera criterio de gravedad según la guía colombiana, excepto la hemoglobina, cuyo valor coincide con el de la OMS.

** Criterio de gravedad según la OMS

gestantes y la mediana fue de $1,02 \mathrm{mg} / \mathrm{dl}(\mathrm{RIC}=0,74-1,67)$; la mediana de la bilirrubina directa entre 35 mujeres gestantes fue de $0,5 \mathrm{mg} / \mathrm{dl}(\mathrm{RIC}=0,31-1,0)$. La bilirrubina total estaba elevada en $21(50,0 \%)$ mujeres gestantes, de las cuales $6(14,2 \%)$ tenían más de 3,0 mg/dl. La bilirrubina directa se encontró elevada (más de $0,3 \mathrm{mg} / \mathrm{dl}$ ) en $27(77,1 \%)$ pacientes y, en 10 casos $(28,6 \%)$, estuvo por encima de $0,9 \mathrm{mg} / \mathrm{dl}$ (alteración grave: $>0,8$ ). De las tres mujeres gestantes con bilirrubina total mayor de $4 \mathrm{mg} / \mathrm{dll}$, dos estaban infectadas por $P$. $\operatorname{vivax}(5,1$ y $4,2 \mathrm{mg} / \mathrm{dl})$ y una por $P$. falciparum $(4,4 \mathrm{mg} / \mathrm{dl})$. No se encontraron diferencias estadísticamente significativas según la especie parasitaria.

La alanina aminotransferasa (ALT) en 20 casos presentó una mediana de $18,6 \mathrm{UI} / \mathrm{L}(\mathrm{RIC}=12,9-38,0)$ y, en $4(20 \%)$ se presentaron valores por encima de $43 \mathrm{UI} / \mathrm{L}$. En dos mujeres gestantes infectadas por $P$. vivax, su valor fue cinco veces superior al rango normal, de 228 y $294 \mathrm{UI} / \mathrm{L}$, respectivamente. La aspartato aminotransferasa (AST) en 20 mujeres embarazadas presentó una mediana de 27,3 UI/L y 6 (30\%) presentaron más de $35 \mathrm{UI} / \mathrm{L}$. Una paciente con $P$. vivax y otra con $P$. falciparum tuvieron cifras con criterio de gravedad: 349 y $127 \mathrm{UI} / \mathrm{L}$, respectivamente.

La glucemia solo se cuantificó en 17 casos y su promedio fue de $93,1 \mathrm{mg} /$ dl $(\mathrm{RIC}=76-101)$. Se diagnosticó hipoglucemia leve a moderada (rango=40-70 $\mathrm{mg} / \mathrm{dl})$ en $3(17,6 \%)$ y ninguna mujer gestante tuvo hipoglucemia grave ( $<40$ $\mathrm{mg} / \mathrm{dl}$ ). No se presentaron diferencias estadísticamente significativas según la especie de plasmodio.

\section{Malaria grave durante el embarazo}

En el cuadro 4 se clasifican cualitativamente los resultados de laboratorio según el grado de alteración, los cuales sirvieron para determinar si los casos eran complicados o no complicados; no se incluyeron el pH sanguíneo ni el bicarbonato plasmático, que solo se midieron en cinco pacientes muy graves. 
En total, se detectaron 26 casos complicados, 15 con alteración grave y 11 con alteración moderada (cuadro 5). No hubo diagnóstico clínico de síndrome de dificultad respiratoria aguda ni de malaria cerebral.

En 15 (13,5\%) de los 111 casos analizados, hubo complicación grave según los criterios de la OMS (15). De estos, 10 (66,7\%) correspondieron a infecciones por $P$. vivax y $5(33,3 \%)$, por $P$. falciparum. Se presentó una sola complicación en 11 (73,3\%) casos y, más de una, en 4 (26,7\%). Las complicaciones diagnosticadas según los criterios de la OMS incluyeron:

- disfunción hepática muy grave en 7 (46,7\%) de los 15 casos, que representan el $6,3 \%$ de todas las pacientes, 6 con aumento de la concentración de la bilirrubina sérica (entre 3,19 y $5,1 \mathrm{mg} / \mathrm{dl}$ ), 2 con AST aumentada (127 y $349 \mathrm{UI} / \mathrm{L}$ ) y 2 con ALT aumentada (228 y $294 \mathrm{UI} / \mathrm{L}$ );

- acidosis en $5(33,3 \%)$ de los 15 casos, 4 con aumento del bicarbonato plasmático mayor de $15 \mathrm{mmol} / \mathrm{L}$ y $1 \mathrm{con} \mathrm{pH}$ sanguíneo de menos de 7,35; solo uno de estos casos cumplió, además, un criterio clínico de alteración neurológica (convulsiones sin cuantificar), en el cual se presentó anemia y trombocitopenia moderadas, e hiperbilirrubinemia grave;

- $\quad$ anemia grave en 4 casos, con valores de hemoglobina entre 3,8 y $6,8 \mathrm{mg} / \mathrm{dl}$, hematocrito entre $11 \%$ y $21 \%$, y concentración de hemoglobina corpuscular media entre 29 y $33 \mathrm{pg}$;

- trombocitopenia grave en 3 casos, con recuento entre 9.300 y 23.400 plaquetas/ $/$ l.

Cuando se sumaron los criterios de lesión moderada y grave (16), consideradas como complicaciones, los casos ascendieron a $26(23,4 \%)$. De estos, $19(73,1 \%)$ fueron infecciones causadas por $P$. vivax, $6(23,1 \%)$, por $P$. falciparum y $1(3,8 \%)$ fue una infección mixta por estas especies. Se diagnosticaron complicaciones en $19(22,3 \%)$ de 85 casos de $P$. vivax, en 6 (37,5\%) de 16 casos de $P$. falciparum y en 1 (25\%) de 4 casos mixtos, sin que ello represente una diferencia estadísticamente significativa.

Cuadro 5. Complicaciones diagnosticadas y valores de laboratorio según el estado de gravedad en 111 mujeres gestantes con malaria en Antioquia

\begin{tabular}{|c|c|c|c|c|c|c|c|}
\hline \multirow[t]{2}{*}{ Complicación } & \multirow[t]{2}{*}{ Examen de laboratorio } & \multicolumn{2}{|r|}{$\begin{array}{c}\text { Sin complicación } \\
n=85\end{array}$} & \multicolumn{2}{|r|}{$\begin{array}{l}\text { Complicación, } \\
\text { guía colombiana1 } \\
n=26\end{array}$} & \multirow{2}{*}{\multicolumn{2}{|c|}{$\begin{array}{l}\text { Complicación, } \\
\text { guía de la } \text { OMS }^{2} \\
\text { n=15 } \\
\text { n Media/(DE)/mediana }\end{array}$}} \\
\hline & & $\mathbf{n}$ & Media/(DE)/mediana & $\mathbf{n}$ & Media/(DE)/mediana & & \\
\hline Anemia $^{3}$ & Hemoglobina (g/dl) & 105 & $10,4(1,8) 10,3$ & 4 & $5,9(1,4) 6,4$ & 4 & $5,9(1,4) 6,4$ \\
\hline Trombocitopenia & Recuento plaquetario (plaquetas/ $\mu \mathrm{l}$ ) & 86 & 179.291 (110.524) 66.000 & 13 & 37.877 (6.656) 39.000 & 3 & $16.133(7.200) 16.000$ \\
\hline Hipoglucemia ${ }^{4}$ & Glucosa (g/dl) & 15 & $96,6(23,5) 91,0$ & 0 & - & 0 & - \\
\hline \multirow[t]{2}{*}{ Disfunción renal ${ }^{5}$} & Nitrógeno ureico (mg/dl) & 21 & $8,9(7,1) 8,0$ & 0 & - & 0 & - \\
\hline & Creatinina (mg/dl) & 35 & $0,7(0,04) 0,6$ & 0 & - & 0 & - \\
\hline \multirow[t]{4}{*}{ Disfunción hepática } & Bilirrubina total (mg/dl) & 30 & $0,8(0,3) 0,9$ & 12 & $3,0(1,4) 2,9$ & 7 & $3,9(1,2) 4,2$ \\
\hline & Bilirrubina directa (mg/dl) & 24 & $0,5-(0,3) 0,4$ & 11 & $1,6(0,9) 1,1$ & 7 & $2,0(1,0) 1,9$ \\
\hline & Aspartato aminotransferasa (UI/L) & 14 & $26,7(12,5) 23,6$ & 6 & $102,2(126,7) 41,5$ & 5 & $118,0(134,0) 48$ \\
\hline & Alanina aminotransferasa (UI/L) & 14 & $24,1(16,7) 17,6$ & 6 & $101,7(125,6) 32,0$ & 6 & $119,4(131,8) 40,1$ \\
\hline
\end{tabular}

${ }^{1}$ Complicación grave, Colombia, incluye los pacientes con criterios de la OMS.

${ }^{2}$ Pacientes que solo cumplían los criterios de gravedad de la OMS.

${ }^{3}$ El criterio de anemia grave de la guía colombiana coincide con el de la guía actual de la OMS.

${ }^{4}$ Hipoglucemia leve en dos casos

${ }^{5}$ Disfunción renal leve en dos casos 
La edad de las mujeres embarazadas no presentó diferencia entre los casos no complicados y los complicados (mediana=21 años, en ambos grupos). La edad gestacional en semanas establecida por ecografía, no presentó diferencia estadística (U de Mann-Whitney, $p>0,05)$ entre los casos complicados (mediana $=29,5$ ) y los no complicados (mediana $=30,2$ ), y tampoco hubo diferencia significativa entre el número de controles prenatales realizados entre los casos complicados (mediana $=3,0$ ) y los no complicados (mediana=4,0) (U de Mann-Whitney, $p>0,05)$. En 12 (38\%) de 32 primigestantes se presentó alguna complicación clínica, en comparación con 14 (18\%) de 79 multigestantes. De los 26 casos complicados, 12 (46,2\%) se presentaron en primigestantes (Odds Ratio, $\mathrm{OR}=2,8$; IC: 1,1-7,0; $p=0,029$ ).

La proporción de casos complicados fue similar para cada trimestre de la gestación. De los 108 casos con edad gestacional calculada, hubo $3(21,4 \%)$ de 14 casos graves que se presentaron en el primer trimestre, 6 (25\%) de 18 en el segundo trimestre y 17 (24,3\%) de 70 casos en el tercer trimestre.

Entre las mujeres embarazadas con antecedente conocido de malaria, las que no presentaron complicación clínica tuvieron más episodios (mediana=2) que las complicadas (mediana $=1$ ), aunque sin diferencia estadísticamente significativa ( $U$ de Mann-Whitney, $p>0,05$ ).

\section{Signos de peligro}

Se encontró por lo menos un signo general de peligro en $44(39,6 \%)$ pacientes. La palidez, aunque no se describe en las historias clínicas como palidez intensa, se incluyó como signo de peligro y fue el más frecuente ( $n=17 ; 15,3 \%$ ), con distribución similar entre las mujeres gestantes con complicación y sin ella. Le siguió la ictericia ( $n=11 ; 10,2 \%)$, que fue más frecuente en los siete casos complicados, con una diferencia significativa (OR=2,28; IC: 1,01-4,9; ji al cuadrado, $p<0,003$ ).

Otros signos menos frecuentes fueron: dolor en la región esplénica $(n=1)$, dolor en la región hepática $(n=2)$, convulsiones $(n=2)$, delirio, letargia o somnolencia $(n=4)$, tos $(n=4)$, anuria u oliguria $(n=5)$, vómito a repetición $(n=7)$ e hiperpirexia $(n=9)$. La presencia de alguno de estos signos se relacionó con una mayor probabilidad de complicación (OR=1,3; IC: 1,0-1,6; ji al cuadrado, $p=0,028)$.

Se presentaron signos clínicos considerados de peligro para la gestación en $45(40,5 \%)$ pacientes; la cefalea persistente fue el más frecuente, seguido del dolor abdominal y el sangrado vaginal (cuadro 6). La presencia de por lo menos uno de estos signos se relacionó con mayores probabilidades de presentar una complicación clínica $(\mathrm{OR}=1,4$; IC: 1,1-1,8; ji al cuadrado, $p<0,003)$. Se reconoció la sintomatología indicativa de preeclampsia en 6 $(5,4 \%)$ de las pacientes, una de las cuales presentó disfunción hepática y trombocitopenia graves.

No se encontraron diferencias estadísticamente significativas de la cefalea ni del dolor abdominal según la especie de plasmodio.

\section{Resultados materno-neonatales}

Durante el episodio de malaria, 55 (49,5\%) mujeres gestantes dieron a luz. La proporción de bajo peso al nacer $(<2.500 \mathrm{~g})$ fue de $29,1 \%(16$ de 55), con un promedio de peso de los neonatos de $2.904 \mathrm{~g}$ (DE=581; mediana=3.020). Por su parte, en el 70,8 \% de los nacimientos el parto fue 
Cuadro 6. Signos de peligro para la gestación en 111 pacientes con malaria en Antioquia, 2010-2014

\begin{tabular}{lcr}
\hline \multicolumn{1}{c}{ Tipo de signo } & $\mathbf{n}$ & \multicolumn{1}{c}{$\%$} \\
\hline Cefalea persistente $_{\text {Dolor abdominal }}^{*}$ & 29 & 26,1 \\
Sangrado vaginal $^{*}$ & 10 & 9,0 \\
Dolor dorsal bajo & 9 & 8,1 \\
Mareos o desmayos & 7 & 6,3 \\
Dolor hipogástrico & 5 & 4,5 \\
Pérdida de líquido por vagina & 5 & 4,5 \\
Ausencia de movimiento fetal & 4 & 4,5 \\
Alteración visual** & 4 & 3,6 \\
Edema de manos o cara & 3 & 2,7 \\
Incremento de presión pélvica $^{*}$ & 2 & 1,8 \\
\hline
\end{tabular}

* No se describió como dolor abdominal persistente.

${ }^{*}$ Visión borrosa $(n=3)$, fotopsias $(n=1)$

prematuro (<36 semanas), con un promedio de edad gestacional de 30 semanas $(\mathrm{DE}=8,8)$. El $32,1 \%$ de los neonatos presentaron una talla inferior a $48 \mathrm{~cm}$ y el 33,3\% presentó un perímetro cefálico por debajo de los $33 \mathrm{~cm}$. No se observaron diferencias estadísticamente significativas en los resultados neonatales, entre los casos complicados y los no complicados (cuadro 7).

Cuadro 7. Resultados materno-neonatales en 55 partos ocurridos durante el episodio de malaria por complicaciones según los criterios de la guía colombiana, Antioquia, 2010-2014

\begin{tabular}{|c|c|c|c|c|c|c|c|}
\hline \multirow{2}{*}{ Resultado } & \multicolumn{2}{|c|}{$\begin{array}{l}\text { Total } \\
n=55\end{array}$} & \multicolumn{2}{|c|}{$\begin{array}{l}\text { Malaria complicada } \\
\qquad n=7\end{array}$} & \multicolumn{2}{|c|}{$\begin{array}{l}\text { Malaria no complicada } \\
n=48\end{array}$} & \multirow[t]{2}{*}{$\mathbf{p}$} \\
\hline & n (\%) & $\mathrm{IC}_{95 \%}{ }^{\dagger}$ & n (\%) & $I C_{95 \%}$ & n (\%) & $\mathrm{IC}_{95 \%}{ }^{\dagger}$ & \\
\hline \multicolumn{8}{|l|}{ Peso al nacer } \\
\hline Bajo $<2.500 \mathrm{~g}$ & $16(29,1)$ & $18,3-42,7$ & $3(42,9)$ & $12,5-79,6$ & $13(27,1)$ & $16,1-41,7$ & $0,402^{*}$ \\
\hline Normal & $39(70,9)$ & $57,2-81,6$ & $4(57,1)$ & $20,3-87,4$ & $35(72,9)$ & $58,2-83,8$ & \\
\hline \multicolumn{8}{|l|}{ Talla neonato } \\
\hline Bajo $<48 \mathrm{~cm}$ & $17(32,1)$ & $20,6-46,1$ & $3(42,9)$ & $12,5-79,7$ & $14(30,4)$ & $18,5-45,5$ & $0,667^{*}$ \\
\hline Normal & $36(67,9)$ & $53,8-79,3$ & $4(57,1)$ & $20,2-87,4$ & $32(69,6)$ & $54,4-81,4$ & \\
\hline \multicolumn{8}{|l|}{ Perímetro cefálico } \\
\hline Bajo <33 cm & $13(33,3)$ & $19,9-50,0$ & $2(50,0)$ & $8,8-91,1$ & $11(31,4)$ & $17,8-49,1$ & $0,589^{*}$ \\
\hline Normal & $26(66,7)$ & $49,9-80,0$ & $2(50,0)$ & $8,8-91,1$ & $24(68,6)$ & $50,8-82,1$ & \\
\hline \multicolumn{8}{|l|}{ Edad gestacional } \\
\hline Bajo $<36$ semanas & $34(70,8)$ & $56,0-82,2$ & $5(71,4)$ & $28,8-93,9$ & $29(70,7)$ & $54,5-82,9$ & $0,673^{*}$ \\
\hline Normal & $14(29,2)$ & $17,7-43,9$ & $2(28,6)$ & $6,0-71,1$ & $12(29,3)$ & $17,0-45,4$ & \\
\hline \multicolumn{8}{|l|}{ Apgar 1} \\
\hline Bajo $<7$ & $1(2,1)$ & $0,2-14,5$ & $1(16,7)$ & $1,7-69,1$ & 0 & - & $0,128^{*}$ \\
\hline Normal & $46(97,9)$ & $85,4-99,7$ & $5(83,3)$ & $30,8-98,2$ & $41(100)$ & - & \\
\hline Peso de neonato en gramos $(M \pm D E)$ & $2.858(671)$ & $2.678-3.037$ & $2.522(646)$ & $1.925-3.120$ & $2.959(556)$ & 2.714-3.097 & ${ }^{1} 0,063^{\dagger}$ \\
\hline Talla en cm (Me $\pm \mathrm{RIC})$ & $48(47-49)$ & - & $48(47-49)$ & - & $48(43-49)$ & - & $0,431^{* *}$ \\
\hline Perímetro cefálico ( $\mathrm{M} \pm \mathrm{DE})$ & $39,2(19,4)$ & $33,2-45,2$ & $45,8(29,8)$ & $8,7-82,8$ & $38,3(18,1)$ & $32,4-44,3$ & $0,784^{+}$ \\
\hline Edad gestacional parto $(\mathrm{M} \pm \mathrm{DE})$ & $30(8,8)$ & $27,5-32,6$ & $30,4(9,3)$ & $21,7-39,1$ & $30,0(8,8)$ & $27,2-32,8$ & 0,91 \\
\hline Apgar $1(\mathrm{Me} \pm \mathrm{RIC})$ & $9(8-9)$ & - & $9(8-9)$ & - & $8,5(8-9)$ & - & $0,652^{* *}$ \\
\hline
\end{tabular}

M: media; Me: mediana; DE: desviación estándar; RIC: rango intercuartílico

* Prueba de ji al cuadrado de independencia. Significación del 0,05

** Prueba U de Mann-Whitney. Significación del 0,05

† Prueba t de Student. Significación del 0,05 


\section{Discusión}

La malaria es un problema importante de salud pública, especialmente en las mujeres gestantes, debido a su asociación con efectos adversos maternos y neonatales.

El cuadro clínico característico de la malaria corresponde a un síndrome febril precedido de escalofríos, el cual se acompaña de síntomas inespecíficos como cefalea, dolor osteomuscular generalizado y debilidad. La progresión a malaria grave está condicionada por diversos factores del parásito y del huésped, y se caracteriza por una falla multisistémica cuyas principales complicaciones incluyen malaria cerebral, edema pulmonar, falla renal aguda, anemia grave, acidosis metabólica e hipoglucemia (7).

Las mujeres gestantes representan un importante grupo de riesgo por tener mayor propensión a adquirir la infección y desarrollar malaria complicada. Las consecuencias de la malaria gestacional más informadas incluyen infección placentaria, anemia materna, bajo peso al nacimiento, parto prematuro, infección congénita e incremento en la mortalidad materno-neonatal $(4,5,18)$.

En este estudio se diagnosticó malaria grave en $23,4 \%$ de las mujeres gestantes estudiadas, y el 13,5\% cumplió con los criterios de gravedad de la OMS. Las complicaciones más frecuentes fueron disfunción hepática, anemia grave, acidosis y trombocitopenia grave. Dos pacientes presentaron convulsiones durante la hospitalización. Sin embargo, no se consideraron como casos de malaria cerebral porque no se conoció la frecuencia de estos episodios; una de ellas presentó disfunción hepática grave y acidosis, y la otra, trombocitopenia grave.

En un estudio previo (14), de 166 mujeres gestantes de Colombia, el $9 \%$ cumplía los criterios de gravedad de la OMS, que incluían disfunción hepática $(5,4 \%)$, anemia grave (2\%), hemoglobinuria y disfunción renal (menos del $1 \%)$.

Debe tenerse presente que la gran frecuencia de complicaciones encontrada en este estudio no refleja la prevalencia de malaria grave durante la gestación, dado que el estudio fue de base hospitalaria en el segundo y el tercer nivel de atención, dejando por fuera los casos atendidos en el primer nivel, que son los casos agudos. De otro lado, dado que en Colombia deben remitirse los casos graves desde el primer nivel, los casos estudiados sí dan cuenta de la presentación clínica y su frecuencia.

En este estudio, la mayoría de las mujeres embarazadas con malaria eran de etnia mestiza, menores de 20 años, multigestantes, y presentaron la malaria durante el tercer trimestre de gestación. Las primigestantes tuvieron una mayor probabilidad de presentar malaria grave que las multigestantes. La edad no difirió entre las pacientes que presentaron alguna complicación $y$ las que no lo hicieron ( $U$ de Mann-Whitney, $p>0,05$ ). Estos resultados coinciden con los de un estudio en Honduras (19), en el cual se encontró que el $82 \%$ de la mujeres embarazadas eran adolescentes (15 a 29 años) y que el $68 \%$ de los casos analizados de malaria en el embarazo ocurrieron durante el tercer trimestre de gestación. Resultados en la misma dirección también fueron reportados en una cohorte de mujeres gestantes de la región de Urabá en Colombia (13).

La asistencia regular al programa de control prenatal, con tres o más visitas en la mitad de casos, puede relacionarse con la detección oportuna de la malaria. Aunque el número de controles en los casos complicados no fue 
inferior al de los no complicados, la evolución hacia un cuadro clínico grave podría explicarse, en parte, porque muchas infecciones tienen baja carga parasitaria y no pueden detectarse con la gota gruesa, que es el método diagnóstico de rutina. Esto permite que la infección progrese y cause un daño crónico, de manera que podría facilitarse la aparición de complicaciones (20). En el noroeste de Colombia, en un estudio con 180 mujeres gestantes, se estableció que las infecciones mixtas submicroscópicas por $P$. vivax y $P$. falciparum se asociaron con bajo peso al nacer y prematuridad (21).

La capacidad de las diferentes técnicas microscópicas para permitir una correcta identificación de Plasmodium spp., se ha cuestionado porque un microscopista con experiencia puede diagnosticar 15 parásitos por microlitro de sangre; no obstante, en el caso de la mujer gestante con malaria, esta sensibilidad es insuficiente, sumado a que hay parasitemias asintomáticas (8). Se sabe que una gran proporción de mujeres gestantes residentes en zonas de alta endemia de África y Asia cursan con infecciones asintomáticas debido a cierto grado de inmunidad adquirida que disminuye las manifestaciones clínicas; no obstante, dichas infecciones pueden causar efectos adversos en las madres y en el feto, y se han relacionado con anemia y bajo peso al nacer (22). En zonas de baja endemia, como en Colombia, la infección asintomática ha sido menos estudiada. Se ha descrito un riesgo 5,4 veces mayor de tener malaria asintomática en mujeres embarazadas, en comparación con las mujeres no gestantes (23); sin embargo, su frecuencia y sus efectos son poco conocidos en este entorno.

En este estudio se encontró que 78,8 \% de las embarazadas presentó fiebre en el momento del diagnóstico de malaria, lo que indica que en alrededor del $21 \%$ de las infecciones no hubo fiebre, situación que refuerza la idea de que deben practicarse pruebas diagnósticas de malaria durante los controles prenatales para favorecer el diagnóstico oportuno. Esta frecuencia de casos afebriles es superior a lo encontrado previamente $(10,2$ $\%$ ) en mujeres gestantes con malaria en el noroeste de Colombia (13). Tal proporción puede considerarse alta en el contexto epidemiológico colombiano, porque otros estudios, aunque en población general, han reportado ausencia de infecciones afebriles en regiones de transmisión activa (24).

No está claramente documentado en las historias si el episodio estudiado fue el primero o una recurrencia de un episodio diagnosticado durante la gestación actual, lo cual aportaría información muy útil para reconocer los casos complicados que se asocian con infección persistente. Solo en 24 casos se conocieron los antecedentes de malaria antes de la gestación, la mayoría por $P$. vivax y ocurridas en el último año, por lo cual la malaria gestacional actual podría atribuirse a una recaída. Aunque sin diferencia estadística, se observó un mayor número de episodios previos de malaria en las no complicadas, lo que está a favor de una mayor tolerancia con manifestaciones clínicas menos graves, como se ha constatado en regiones de alta endemia (25).

Una condición que posiblemente contribuye a la prevención de casos graves, es que las mujeres gestantes fueron atendidas en la primera semana del inicio de la enfermedad, $59 \%$ durante los tres primeros días. Esta situación se explica por el cumplimiento, en el primer nivel de atención, de remitir desde los puestos de malaria a un centro hospitalario a toda mujer gestante con examen positivo y, desde allí, hacia el segundo nivel todo caso con sospecha o confirmación de gravedad. 
La clasificación de los casos graves realizada por los médicos mediante la CIE-10, indica que la mayoría de los casos complicados no se reconocen como tales; solo tres casos fueron correctamente clasificados como graves entre los 26 determinados mediante los exámenes de laboratorio. Esto, sumado a que no se maneja un protocolo de atención para la malaria gestacional, puede explicar que en muchos casos no se soliciten exámenes esenciales para detectar lesiones orgánicas, como el de creatinina, nitrógeno ureico, glucemia y transaminasas, lo que impide conocer de manera completa las complicaciones producidas. Incluso, en algunos casos, no se registró información esencial como la edad gestacional durante la consulta o en el parto.

Las mujeres embarazadas con malaria presentan complicaciones con mayor frecuencia. En regiones de baja endemia se estima que hay un mayor riesgo durante el segundo y el tercer trimestres de gestación, especialmente debido a la hipoglucemia y al síndrome de dificultad respiratoria (26). En este estudio se encontró que la proporción de complicaciones fue similar en los diferentes trimestres de la gestación, aunque la mayoría de los casos de malaria $(65 \%)$ se diagnosticó durante el tercer trimestre. Dado que la infección puede adquirirse antes de la gestación y manifestarse durante el embarazo (27), no es posible saber si se adquirió en un trimestre en particular o si ya estaba presente antes de iniciado el embarazo, situación que podría explicar la mayor o menor predisposición a sufrir un cuadro clínico grave.

La presencia de signos generales de peligro se asoció con la gravedad clínica, entre ellos la ictericia, que ha demostrado ser un buen predictor de complicación (28). Además, se observaron otros signos clínicos considerados de alarma durante la gestación en $40 \%$ de las pacientes, los cuales se relacionaron con gravedad; los más frecuentes fueron cefalea persistente, dolor abdominal y sangrado vaginal. Estos signos, además de anunciar una complicación obstétrica, pueden indicar una preeclampsia, condición que fue diagnosticada en $5,4 \%$ de los casos estudiados y la cual se ha considerado que puede asociarse con la malaria (26).

Aunque con resultados más desfavorables en los neonatos nacidos de las mujeres gestantes con malaria grave, los efectos observados no son estadísticamente diferentes de aquellos de los neonatos nacidos de las madres sin complicación, posiblemente porque solo siete de aquellas tuvieron el parto durante su hospitalización y de los demás casos complicados no se tuvo información sobre el parto.

El estudio contiene sesgos inherentes a la falta de una medición estandarizada de la condición clínica de las pacientes y de los resultados, y a la no inclusión de las mujeres embarazadas hospitalizadas en el primer nivel de atención, aunque se espera que estos sean todos cuadros clínicos agudos, dado que existe la instrucción de remitir aquellos que muestren deterioro clínico.

La falta de información estadística a nivel nacional y departamental sobre la condición en estudio, no permitió constatar si se incluyeron razonablemente los casos diagnosticados durante el período de estudio, lo que puede constituirse en una limitación de la investigación.

Este estudio da cuenta de los casos de malaria gestacional hospitalizados durante cinco años en el departamento de Antioquia, el cual ocupa el tercer lugar en Colombia en casos de malaria. Se llevó a cabo en tres hospitales de tercer nivel de la capital a donde se remiten casos graves, y en tres de los 
principales hospitales de segundo nivel localizados en las regiones endémicas, razón por la cual puede afirmarse que los casos estudiados reflejan la situación de la malaria gestacional grave y su diagnóstico en el departamento.

Permitió establecer los casos graves de malaria en mujeres embarazadas que alcanzaron a recibir atención hospitalaria de segundo y tercer nivel de complejidad, la mayoría de manera oportuna, lo que pudo haber favorecido la reducción de las complicaciones clínicas.

Se propone elaborar un protocolo para la atención hospitalaria de las mujeres embarazadas con malaria para precisar el estado clínico y lograr el registro correcto de los diagnósticos que permita un seguimiento adecuado de la paciente e, incluso, del neonato.

\section{Agradecimientos}

Al Hospital Universitario San Vicente Fundación, al Hospital Pablo Tobón Uribe y al Hospital General de Medellín, en Medellín; al E.S.E. Hospital César Uribe Piedrahita, en el municipio de Caucasia; al Hospital Antonio Roldán Betancur, en el municipio de Apartadó, y al Hospital La Cruz E.S.E., en el municipio de Puerto Berrío, por su aval para el acceso a las historias clínicas. A los profesores de la Universidad de Antioquia, Jesús Velásquez y Juan Gabriel Piñeros, por su acompañamiento académico en la ejecución del estudio.

\section{Referencias}

1. World Health Organization. World malaria report, 2015. Geneva. WHO; 2016. Fecha de consulta: 5 de marzo de 2018. Disponible en: http://apps.who.int/iris/bitstream/ handle/10665/205559/WHO_HTM_GMP_2016.2_spa.pdf;jsessionid=DD8C013034924EE5 A4599CEE0C450615? sequence $=1$

2. Dellicour S, Tatem AJ, Guerra CA, Snow RW, ter Kuile FO. Quantifying the number of pregnancies at risk of malaria in 2007: A demographic study. PLoS Med. 2010;7:e1000221. https://doi.org/10.1371/journal.pmed.1000221

3. Menéndez C, Mayor A. Congenital malaria: The least known consequence of malaria in pregnancy. Semin Fetal Neonatal Med. 2007:12:207-13.

https://doi.org/10.1016/j.siny.2007.01.018

4. Desai M, ter Kuile FO, Nosten F, McGready R, Asamoa K, Brabin B, et al. Epidemiology and burden of malaria in pregnancy. Lancet Infect Dis. 2007;7:93-104. https://doi.org/10.1016/S1473-3099(07)70021-X

5. Steketee RW, Nahlen BL, Parise ME, Menéndez C. The burden of malaria in pregnancy in malaria-endemic areas. Am J Trop Med Hyg. 2001;64(Suppl.1):28-35.

6. Geertruyden JV, Ntakirutimana D, Erhart A, Rwagacondo C, Kabano A, D’Alessandro U. Malaria infection among pregnant women attending antenatal clinics in six Rwandan districts. Trop Med Int Health. 2005;10:681-8. https://doi.org/10.1111/j.1365-3156.2005.01431.x

7. World Health Organization. Severe falciparum malaria. Trans R Soc Trop Med Hyg. 2000;94(Suppl.1):1-90.

8. Nosten F, Rogerson SJ, Beeson JG, McGready R, Mutabingwa TK, Brabin B. Malaria in pregnancy and the endemicity spectrum: What can we learn? Trends Parasitol. 2004;20:425-32. https://doi.org/10.1016/j.pt.2004.06.007

9. Greenwood B, Alonso P, ter Kuile FO, Hill J, Steketee RW. Malaria in pregnancy: Priorities for research. Lancet Infect Dis. 2007;7:169-74. https://doi.org/10.1016/S1473-3099(07)70028-2

10. Ramal C, Pinedo P. Malaria en gestantes entre marzo del 2002 y julio del 2003: experiencia en el Hospital Regional de Loreto, Perú. Acta Med Peruana. 2008;25:220-3.

11. Chagas EC, do Nascimento CT, de Santana Filho FS, Bôtto-Menezes CH, MartínezEspinosa FE. Impact of malaria during pregnancy in the Amazon region. Rev Panam Salud Pública. 2009;26:203-8. 
12. Piñeros JG. Epidemiología de la malaria durante el embarazo. Med UIS. 2008;21:142-57.

13. Piñeros JG, Tobón A, Álvarez G, Portilla C, Blair S. Maternal clinical findings in malaria in pregnancy in a region of northwestern Colombia. Am J Trop Med Hyg. 2013;89:520-6. https://doi.org/10.4269/ajtmh.12-0205

14. Dirección Seccional de Salud, Departamento de Antioquia. Indicadores básicos de salud 2014. Fecha de consulta: 5 de marzo de 2018. Disponible en: http://diagnosticosalud.dssa.gov.co/\#

15. World Health Organization. Tratamiento del paludismo grave. Manual Práctico. $3^{\text {a }}$ edición. Ginebra: WHO; 2014. Fecha de consulta: 5 de abril de 2018. Disponible en: http://apps.who.int/medicinedocs/documents/s20170es/s20170es.pdf

16. Organización Panamericana de la Salud. Guía para atención clínica integral del paciente con malaria. Bogotá: Ministerio de Protección Social; 2010. Fecha de consulta: 23 de marzo de 2018. Disponible en: https://www.paho.org/coL/index.php?option=com

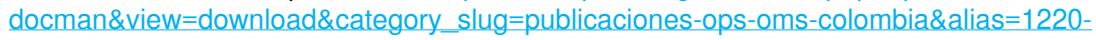
guia-para-la-atencion-clinica-integral-del-paciente-con-malaria\&ltemid=688

17. Tobón A. Signos de peligro en el paciente con malaria. Biomédica. 2009;29:320-9. https://doi.org/10.7705/biomedica.v29i2.33

18. Menéndez C, D'Alessandro U, ter Kuile FO. Reducing the burden of malaria in pregnancy by preventive strategies. Lancet Infect Dis. 2007;7:126-35. https://doi.org/10.1016/S1473-3099(07)70024-5

19. Fernández RD, García Y, Alger J. Malaria y embarazo: observaciones clínicoepidemiológicas en dos zonas geográficas de Honduras. Rev Med Hondur. 2001;69:8-18.

20. Cottrell G, Moussiliou A, Luty AJ, Cot M, Fievet N, Massougbodji A, et al. Submicroscopic Plasmodium falciparum infections are associated with maternal anemia, premature births, and low birth weight. Clin Infect Dis. 2015;60:1481-8. https://doi.org/10.1093/cid/civ122

21. Gavina K, Gnidehou S, Arango E, Hamel-Martineau C, Mitran C, Agudelo O, et al. Clinical outcomes of submicroscopic infections and correlates of protection of VAR2CSA antibodies in a longitudinal study of pregnant women in Colombia. Infect Immun. 2018;86. https://doi.org/10.1128/IAl.00797-17.

22. Uneke CJ. Impact of placental Plasmodium falciparum malaria on pregnancy and perinatal outcome in sub-Saharan Africa: I: Introduction to placental malaria. Yale J Biol Med. 2007;80:39-50.

23. Khan WA, Galagan SR, Prue CS, Khyang J, Ahmed S, Ram M, et al. Asymptomatic Plasmodium falciparum malaria in pregnant women in the Chittagong Hill Districts of Bangladesh. PLoS One. 2014;9:e98442. https://doi.org/10.1371/journal.pone.0098442

24. Osorio L, Todd J, Bradley D. Ausencia de malaria asintomática en escolares de Quibdó, Chocó. Biomédica. 2004;24:13-9. https://doi.org/10.7705/biomedica.v24i1.1244

25. Galatas B, Bassat Q, Mayor A. Malaria parasites in the asymptomatic: Looking for the hay in the haystack. Trends Parasitol. 2016;32:296-308. https://doi.org/10.1016/j.pt.2015.11.015

26. World Health Organization. Guidelines for the treatment of malaria. Geneva: WHO; 2015. Fecha de consulta: 19 de marzo de 2018. Disponible en: http://apps.who.int/iris/bitstream/handle/10665/162441/9789241549127 eng.pdf? sequence=1

27. Berry I, Walker P, Tagbor H, Bojang K, Coulibaly SO, Kayentao K, et al. Seasonal dynamics of malaria in pregnancy in West Africa: Evidence for carriage of infections acquired before pregnancy until first contact with antenatal care. Am J Trop Med Hyg. 2018;98:534-542. doi: https://doi.org/10.4269/ajtmh.17-0620

28. Tobón A, Giraldo-Castro C, Blair S. Utilidad de los signos clínicos y parasitológicos en el pronóstico de la malaria grave en Colombia. Biomédica. 2012;32:79-94. https://doi.org/10.7705/biomedica.v32i0.613 